\title{
Endmember Extraction from Hyperspectral Image Based on Discrete Firefly
} Algorithm (EE-DFA)

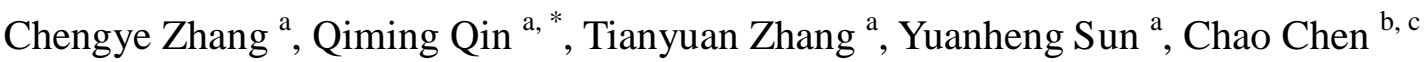 \\ ${ }^{a}$ Institute of Remote Sensing and Geographic Information System, School of Earth and Space Sciences, Peking \\ University, Beijing 100871, China. \\ ${ }^{\mathrm{b}}$ Marine Science and Technology College, Zhejiang Ocean University, Zhoushan, Zhejiang 316022, China. \\ ${ }^{\mathrm{c}}$ Marine Acoustics and Remote Sensing Laboratory, Zhejiang Ocean University, Zhoushan, Zhejiang 316022,
} China.

Corresponding author: qmqin@pku.edu.cn, qmqinpku@163.com

First author: zhangchengye@pku.edu.cn

\section{Abstract}

This study proposed a novel method to extract endmembers from hyperspectral image based on discrete firefly algorithm (EE-DFA). Endmembers are the input of many spectral unmixing algorithms. Hence, in this paper, endmember extraction from hyperspectral image is regarded as a combinational optimization problem to get best spectral unmixing results, which can be solved by the discrete firefly algorithm. Two series of experiments were conducted on the synthetic hyperspectral datasets with different SNR and the AVIRIS Cuprite dataset, respectively. The experimental results were compared with the endmembers extracted by four popular methods: the sequential maximum angle convex cone (SMACC), N-FINDR, Vertex Component Analysis (VCA), and Minimum Volume Constrained Nonnegative Matrix Factorization (MVC-NMF). What's more, the effect of the parameters in the proposed method was tested on both synthetic hyperspectral datasets and AVIRIS Cuprite dataset, and the recommended parameters setting was proposed. The results in this study demonstrated that the proposed EE-DFA method showed better performance than the existing popular methods. Moreover, EE-DFA is robust under different SNR conditions.

\section{Key words}

Endmember Extraction, Discrete Firefly Algorithm (DFA), Hyperspectral Image, Remote Sensing 


\section{Introduction}

Hyperspectral remote sensing acquires image covering hundreds of narrow contiguous spectral bands, which provides a contiguous spectrum for each pixel [1]. However, due to the relative low spatial resolution of hyperspectral image, it is inevitable that several materials are presented together in one pixel in natural environment. Thus, it has triggered a popular research interest to determine the abundance fraction of each material in a mixed pixel from hyperspectral image, which called spectral unmixing. The first step for solving this problem (spectral unmixing) is usually endmember extraction (EE), which is to determine the idealized pure spectral signature of each material. In fact, an endmember extracted from a real hyperspectral image is generally still a mixed pixel that contains high fraction of one kind of material [2]. In computer science and mathematics, discrete optimization is a topic on finding a best object from a finite set of objects. In detail, discrete optimization problem is to find a combination of discrete variables from all feasible solutions to optimize the objective function. What's more, endmember extraction from a hyperspectral image is to find some pixels and combine them as pure spectral signatures of the materials in the image to get the best accuracy of spectral unmixing results. In this sense, endmember extraction from hyperspectral image can be transferred into a typical discrete optimization problem that is of particular importance for spectral unmixing of hyperspectral image.

For recent more than two decades, many methods for endmember extraction from hyperspectral image have been proposed. Typical algorithms developed for endmember extraction include pixel purity index (PPI) [3], [4], N-FINDR [5], iterative error analysis (IEA) [6], vertex component analysis (VCA) [7], the sequential maximum angle convex cone (SMACC) [8], the optical real-time adaptive spectral identification system (ORASIS) [9], convex cone analysis (CCA) [10], automated morphological endmember extraction (AMEE) [11], iterated constrained endmembers (ICE) [12], the single individual evolutionary (SIE) strategy [13]. In addition, some new methods for endmember extraction were also proposed in recent years [14-23]. In fact, currently, although there are a variety of methods for endmember extraction from hyperspectral image, these methods have respective shortcomings. Comparative analysis and discussions on the existing endmember extraction algorithms have been conducted by several scholars [2], [19], [24]. In this paper, disadvantages of several typical algorithms are displayed as examples. For instance, the PPI algorithm is a typical manual/interactive endmember 
extraction method, which is slow and artificially influenced [2]. In the N-FINDR algorithm, it is generally inevitable to reduce the dimensions of the hyperspectral dataset [5]. What's more, many algorithms including the N-FINDR algorithm, VCA algorithm, SMACC algorithm etc., are based on a simple geometrical precondition that the endmembers are the vertexes of a convex simplex in spectral space [5], [7], [8]. However, due to some non-linear factors and the noise in a real hyperspectral image, this precondition is not reliable [7]. Thus, the existing algorithms are still limited and it is necessary to propose new algorithms with particular advantages to conduct endmember extraction from hyperspectral image and provide more optional methods for endmember extraction for scholars in the area of hyperspectral remote sensing.

Firefly algorithm (FA) is a novel nature-inspired algorithm, which is inspired by social behavior of fireflies and was presented by Xin-She Yang [25]. It has been proven that FA is an efficient method to solve optimization problem and is better than both particle swarm optimization (PSO) algorithm and genetic algorithm (GA) in the respects of both efficiency and success rate [26], [27]. Moreover, Ref. [27] has demonstrated that discrete firefly algorithm (DFA) performed better than the ant colony optimization (ACO) algorithm in solving discrete optimization problems. Hence, DFA provides a better way for solving discrete optimization problems including endmember extraction from hyperspectral image, than other popular swarm intelligence algorithms, such as PSO, GA and ACO.

This paper proposes an endmember extraction method from hyperspectral image based on discrete firefly algorithm (EE-DFA). To the best of our knowledge, there is little information available in published literature about the application of firefly algorithm on endmember extraction. To realize the endmember extraction, we redefined the objective function and several variables in FA, and proposed a new discretization method of the firefly algorithm.

\section{Firefly Algorithm (FA)}

A brief introduction to FA is presented here, with the purpose to define necessary concepts to explain the EE-DFA. More details of FA can be found in the Ref. [28]. Nature-inspired algorithms are among the most important and powerful methods for optimization problems. In the nature, most firefly species produce short and rhythmic flashes that is to act as a signal system to attract other fireflies. Therefore, FA obeys the following rules: (1) All fireflies are attractive to other fireflies regardless of their 
sex; (2) the attractiveness of a firefly is determined by its brightness, which means that for any two flashing fireflies the less bright one will move towards the brighter one. If a particular firefly is the brightest in the firefly population, it will move randomly; and (3) the brightness of a firefly is determined by the objective function. For a minimization problem in this study, the brightness of a firefly should be inversely proportional to the value of the objective function [27].

Distance: In FA, the distance $r_{i j}$ of two fireflies $\mathbf{x}_{\mathbf{i}}$ and $\mathbf{x}_{\mathbf{j}}$ can be determined as follows.

$$
r_{i j}=\sqrt{\sum_{k=1}^{n}\left(x_{i k}-x_{j k}\right)^{2}}
$$

where $x_{i k}$ is the $k$-th component of the $i$-th firefly $\mathbf{x}_{\mathbf{i}}$ and $n$ is the number of components of the firefly $\mathbf{x}_{\mathbf{i}}$. Here we give an explanation on the word 'component'. 'component' is used to express a dimension of a vector. For example, if there is a vector $\boldsymbol{V}=(a, b, c, d, e$, $f)$, we could say that the vector $\boldsymbol{V}$ possesses 6 components and " $d$ " is the $4^{\text {th }}$ component of the vector $\boldsymbol{V}$.

Attractiveness: The attractiveness $\beta_{i j}$ between two fireflies $\mathbf{x}_{\mathbf{i}}$ and $\mathbf{x}_{\mathbf{j}}$ is a monotonically decreasing function. For instance, it can be the following form.

$$
\beta_{i j}=\beta_{0} e^{-\gamma r_{i j}^{2}}
$$

where $r_{i j}$ is the distance between two fireflies $\mathbf{x}_{\mathbf{i}}$ and $\mathbf{x}_{\mathbf{j}}, \beta_{0}$ is the attractiveness at $r_{i j}=0$ and $\gamma$ is a fixed light absorption coefficient.

Movement: The movement of the $i$-th firefly $\mathbf{x}_{\mathbf{i}}$, when it is attracted by another brighter one $\left(\mathbf{x}_{\mathbf{j}}\right)$, can be determined as follows.

$$
x_{i k}=x_{i k}+\beta_{0} e^{-\gamma_{i j}^{2}}\left(x_{j k}-x_{i k}\right)+\alpha(\text { rand }-0.5)
$$

where the second term is for the attraction between two fireflies, and the third term is randomization with $\alpha$ being the randomization step and "rand" is a random number uniformly distributed in $[0,1]$. Equation (3) takes $x_{i k}$ as an example to illustrate the calculation method of the components of $\mathbf{x}_{\mathbf{i}}$ after one movement. All the components of $\mathbf{x}_{\mathbf{i}}$ should be calculated according to equation (3) in one movement of $\mathbf{x}_{\mathbf{i}}$.

To apply the FA on endmember extraction, we redefined the inputs, outputs, objective function, the meaning of fireflies, and proposed discretization method. 


\subsection{Definition of the parameters and variables}

In this section, the meaning of all the parameters and variables in EE-DFA is presented. $m$ : Number of endmembers that will be extracted.

$n$ : Number of candidate endmembers and also the number of components of the firefly $\mathbf{x}_{\mathbf{i}}$.

$N$ : Number of the pixels in one spectral band.

$L$ : Number of spectral bands in hyperspectral dataset.

$t: t=0$ means the $i$-th firefly is the current best solution in all fireflies. Otherwise, the $i$-th firefly is not the current best solution in all fireflies.

$\mathbf{p}_{\mathbf{i}}=\left(p_{i 1}, p_{i 2}, p_{i 3}, \ldots, p_{i L}\right), i=1,2,3, \ldots, N$ : The spectral reflectance of the $i$-th pixel.

$\mathbf{p}_{\mathbf{i}}{ }^{\prime}=\left(p^{\prime}{ }_{i 1}, p^{\prime}{ }_{i 2}, p^{\prime}{ }_{i 3}, \ldots, p^{\prime}{ }_{i L}\right), i=1,2,3, \ldots, N$ : The approximate estimation of $\mathbf{p}_{\mathbf{i}}$.

$\mathbf{e}_{\mathbf{j}}=\left(e_{j 1}, e_{j 2}, e_{j 3}, \ldots, e_{j L}\right), j=1,2,3, \ldots, m$ : The spectral reflectance of the $j$-th endmember.

$a_{i j}, i=1,2,3, \ldots, N, j=1,2,3, \ldots, m$ : The abundance fraction of the $j$-th endmember in the $i$-th pixel.

$a_{i j}, i=1,2,3, \ldots, N, j=1,2,3, \ldots, m$ : The least square estimation of $a_{i j}$.

$\mathbf{x}_{\mathbf{i}}=\left(x_{i 1}, x_{i 2}, x_{i 3}, \ldots, x_{i k}, \ldots, x_{i n}\right), x_{i 1+} x_{i 2+} x_{i 3+\ldots+} x_{i n}=m, x_{i k} \in\{0,1\}$ : The $i$-th firefly, also a feasible solution.

$x_{i k}=1$ means that the $k$-th candidate endmember is selected, otherwise is not.

I(i): The brightness of the $i$-th firefly $\boldsymbol{x}_{\boldsymbol{i}}$.

xbest: The firefly that is the best solution.

124 Ibest: The brightness of the firefly that is the best solution.

$\mathbf{Y}=\left\{\left(y_{1}, y_{2}, y_{3}, y_{4} \ldots . . y_{n}\right) \mid y_{1}+y_{2}+y_{3}, \ldots,+y_{n}=m, y_{i} \in\{0,1\}\right\}:$ A feasible solution space including all the feasible solutions.

\subsection{Determination of the objective function}

In the linear spectral mixture analysis (LSMA), the abundance fraction of the $j$-th endmember in the $i$-th pixel $\left(a_{i j}\right)$ obeys to the following equations (4) - (6) [29].

$$
\begin{gathered}
\mathbf{p}_{\mathbf{i}}=\sum_{j=1}^{m} a_{i j} \mathbf{e}_{\mathbf{j}}+{ }_{\mathbf{i}} \\
\sum_{j=1}^{m} a_{i j}=1, \forall i
\end{gathered}
$$




$$
a_{i j} \geq 0, \forall i, \forall j
$$

The full constraints least square (FCLS) is a method to obtain the abundance fraction $a_{i j}$, which obeys to all the equations (4) - (6) [29]. When all the endmembers $\left(\boldsymbol{e}_{j}, j=1,2,3, \ldots, m\right)$ are determined, based on the FCLS, $a_{i j}$ (the least square estimation of $a_{i j}$ ) can be acquired, which is the process of spectral unmixing. $\mathbf{p}_{\mathbf{i}}^{\prime}$ (the estimation of $\mathbf{p}_{\mathbf{i}}$ ) can be obtained according to the following equation.

$$
\mathbf{p}_{\mathbf{i}}{ }^{\prime}=\sum_{j=1}^{m} a_{i j}{ }^{\prime} \mathbf{e}_{\mathbf{j}}
$$

As already mentioned in the introduction section, endmember extraction from a hyperspectral image is to find some pixels as pure spectral signatures of materials to get the best spectral unmixing results. The spectral unmixing results can be evaluated by the root-mean-square error (RMSE) between the real reflectance $\mathbf{p}_{\mathbf{i}}$ and its estimation $\mathbf{p}_{\mathbf{i}}{ }^{\prime}$, which can be calculated according to the following equation.

$$
R M S E=\frac{1}{N} \sum_{i=1}^{N} \sqrt{\frac{1}{L} \sum_{j=1}^{L}\left(p_{i j}^{\prime}-p_{i j}\right)^{2}}
$$

A less RMSE means a better result of spectral unmixing and also endmember extraction. Thus, the brightness $I(\mathbf{x})$ of the firefly $\mathbf{x}$ was defined as the opposite value of the RMSE.

$$
I(\mathbf{x})=-R M S E
$$

Hence the problem of endmember extraction from hyperspectral image is to find the firefly $\mathbf{x}$ that corresponds to the maximum brightness $I(\mathbf{x})$, which could be formulated in a mathematical way as follows:

$$
\begin{aligned}
& \text { maximize } I(\mathbf{x}) \\
& \text { subject to } \mathbf{x} \in \mathbf{Y}
\end{aligned}
$$

\subsection{Discretization of FA}

The $i$-th firefly $\mathbf{x}_{\mathbf{i}}\left(\mathbf{x}_{\mathbf{i}}=\left(x_{i 1}, x_{i 2}, x_{i 3}, \ldots, x_{i k}, \ldots, x_{i n}\right), x_{i 1+}+x_{i 2+} x_{i 3+\ldots+} x_{i n}=m, x_{i k} \in\{0,1\}\right)$ represents a feasible solution for endmember extraction. $x_{i k}=1$ means that the $k$-th candidate endmember is selected, otherwise is not. When the $i$-th firefly moves toward the $j$-th firefly, the position of the $i$-th firefly is recalculated based on (3) and thus changed from a binary number to a real number. To translate this real number to a binary number, this paper proposes a discretization method: the first $m$ largest components of $\mathbf{x}_{\mathbf{i}}$ is defined as 1 and other components is defined as 0 . Here we give an example to illustrate this 
150

discretization method, when $m=7$.

Before discretization: $\mathbf{x}_{\mathbf{i}}=(1.0294,1.0116,0.2540,1.1268,1.2647,0.1951,0.2570,1.0938,1.1150$, 1.0298, 0.3152, 1.0412, 1.1143, 0.9708, 1.1006, 0.2838, 0.8435, 1.0315, 1.0844). The first seven (Because $m$ equals 7) largest components of $\mathbf{x}_{\mathbf{i}}$ is 1.1268, 1.2647, 1.0938, 1.1150, 1.1143, 1.1006 and 1.0844 .

Hence, after discretization: $\mathbf{x}_{\mathbf{i}}=(0,0,0,1,1,0,0,1,1,0,0,0,1,0,1,0,0,0,1)$. This example is just used to explain the proposed discretization method and has nothing to do with the experimental data in this paper.

\subsection{Process of the proposed EE-DFA method}

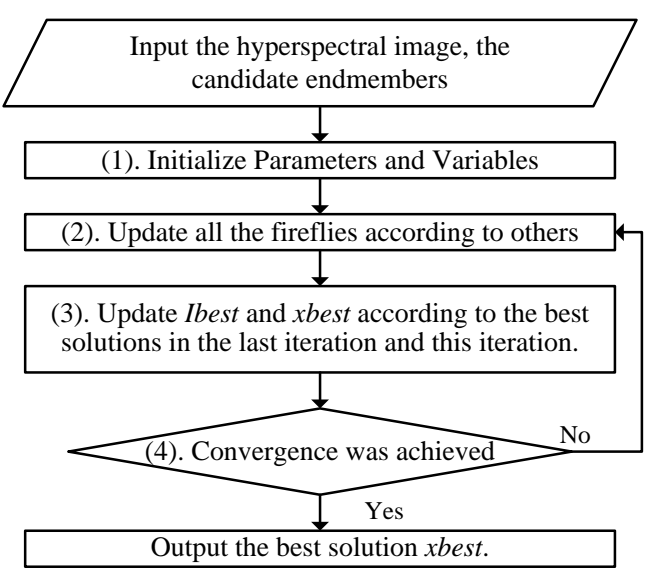

Fig. 1 The flow chart of the procedure of the proposed EE-DFA

The main steps of the proposed EE-DFA method is presented in Fig. 1. The key processes are described as follows.

(1). The parameters and variables that need to be initialized include $\beta_{0}, \gamma, \alpha$, FireflyNumber (the number of fireflies, see the Section 4.2), Fireflies and their brightness. The analysis on the initialization of $\beta_{0}, \gamma$, and $\alpha$ could be found in the Section 4.3. According to the definition of fireflies, for the initialization of the firefly $\mathbf{x}_{\mathbf{i}}$, we randomly set $m$ components as 1 and other components as 0 . The brightness $\mathbf{I}\left(\mathbf{x}_{\mathbf{i}}\right)$ could be calculated based on FCLS and Equation (7) (9).

(2). The implementation method of this step is shown as follows.

for $i=1$ : FireflyNumber

for $j=1$ : FireflyNumber

if $\left(\mathrm{I}\left(\mathbf{x}_{\mathbf{j}}\right)>\mathrm{I}\left(\mathbf{x}_{\mathbf{i}}\right)\right)$ 

199
Move $\mathbf{x}_{\mathbf{i}}$ to $\mathbf{x}_{\mathbf{j}}$ and update $\mathrm{I}\left(\mathbf{x}_{\mathbf{i}}\right)$

end if

end for $j$

if $\mathbf{x}_{\mathbf{i}}$ is the brightest firefly

Move $\mathbf{x}_{\mathbf{i}}$ randomly and update $\mathrm{I}\left(\mathbf{x}_{\mathbf{i}}\right)$

end if

\section{Update Ibest and xbest}

end for $i$

(3). In every iteration, Ibest and xbest should be recorded. In this step, we update Ibest and xbest according to the best solutions in the last iteration and this iteration.

(4). In this study, the condition that judges if the convergence was achieved has been analyzed in the Section 4.3.

\section{Experimental Results and Discussion}

\subsection{Datasets used for experiments}

To evaluate the EE-DFA algorithm proposed in this paper, we applied the proposed algorithm on two series of hyperspectral datasets (synthetic hyperspectral datasets with different SNR and the AVIRIS hyperspectral dataset) for endmember extraction.

\subsubsection{Synthetic hyperspectral datasets}

The synthetic hyperspectral image of size $64 \times 64$ pixels in this study was created by the six standard spectra (Fig. 2) selected from the United States Geological Survey (USGS) spectral library.

First, the six standard spectra (idealized endmembers) were resampled to generate the $10 \mathrm{~nm}$ spectral resolution with the 224 spectral bands covering wavelength-range from $\sim 400 \mathrm{~nm}$ to $2500 \mathrm{~nm}$. Second, the whole image $(64 \times 64)$ was divided into units of $8 \times 8$ blocks. Pixels in each block were filled up by one spectrum randomly selected from the six standard spectra. To generate a linear mixture, a $9 \times 9$ averaging filter was applied on the image. Third, to simulate the noise in the data, we add zero-mean Gaussian noise to the mixture image. The covariance matrix of the added noise was defined as a diagonal matrix $\sigma^{2} \mathbf{A}$ (A was an identity matrix). The Signal-to-Noise Ratio (SNR) was defined as follows.

$$
\mathrm{SNR}=10 \lg \left(\mathrm{E}\left[\mathbf{p}^{\mathrm{T}} \mathbf{p}\right] / \mathrm{E}\left[\boldsymbol{\varepsilon}^{\mathrm{T}} \boldsymbol{\varepsilon}\right]\right)
$$


$\mathbf{p}$ and $\boldsymbol{\varepsilon}$ represented the spectral signature without noise and the noise of a pixel, respectively. E[]

202

203

204

205

206

207

208

209

210

211

212

213

214

215

216

217 was the mathematical expectation in spatial dimension. Thus, when the SNR was given, the noise variance was determined by the equation (12).

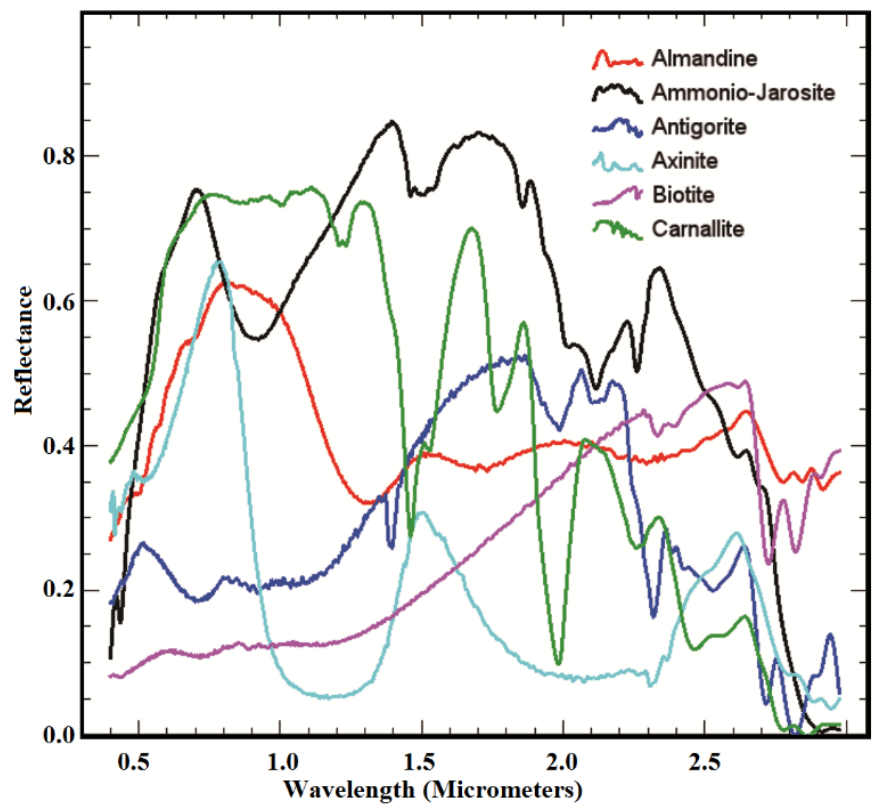

Fig. 2. Six selected spectra for the synthetic hyperspectral dataset.

$$
\sigma^{2}=\mathrm{E}\left[\mathbf{p}^{\mathrm{T}} \mathbf{p}\right] /\left(10^{\mathrm{SNR} / 10} \times \mathrm{L}\right)
$$

The similar creation methods of synthetic hyperspectral datasets were also used in Ref. [15], [30]. To evaluate the impact of different noise levels on EE-DFA method, four synthetic hyperspectral datasets were created with $S N R=50 \mathrm{~dB}, 40 \mathrm{~dB}, 30 \mathrm{~dB}, 20 \mathrm{~dB}$, respectively (Fig. 3). Different image quality with different SNR can be easily found from Fig. 3 by visual investigation.

\subsubsection{The real hyperspectral image}

The hyperspectral dataset acquired by the AVIRIS sensor over Cuprite, Nevada in the USA, was utilized as the real hyperspectral image to test the proposed method in this study. This dataset has been widely used to test new algorithms of endmember extraction and spectral unmixing in hyperspectral remote sensing [2], [21]. In this paper, a 50-band (1990.8 nm - $2479.0 \mathrm{~nm})$ subset (400×350 pixels) of this dataset is used, which is affiliated to the data resource provided by the ENVI software (filename: 
“cup95eff.int”). Fig. 4 shows the false color composition of the used subset.

In this study, the number of endmembers was determined by a method termed hyperspectral signal identification by minimum error (HySIME). By HySIME, the number of synthetic hyperspectral images and real hyperspectral image was determined as 6 and 8, respectively. The first step of this method is to estimate the signal and noise correlation matrices by multiple regression. Then, the subset of eigenvalues was selected to represent the signal subspace in the sense of least squared error, which is calculated by minimizing the sum of the power of projection error with that of noise. More technical details could be found in Reference [31]. This method has been widely used to determine the intrinsic dimensionality of hyperspectral data (e.g., Reference [32] [35]).

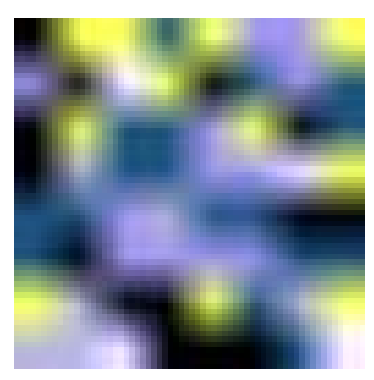

$\mathrm{SNR}=50 \mathrm{~dB}$

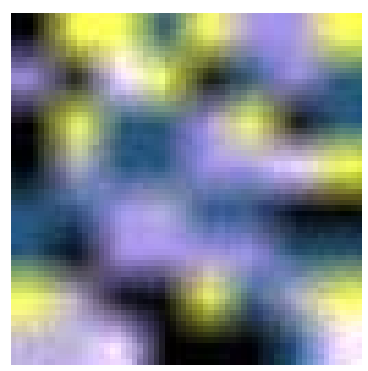

$\mathrm{SNR}=30 \mathrm{~dB}$

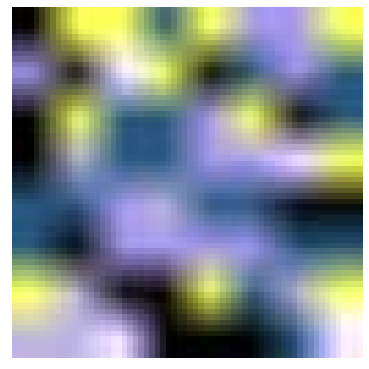

$\mathrm{SNR}=40 \mathrm{~dB}$

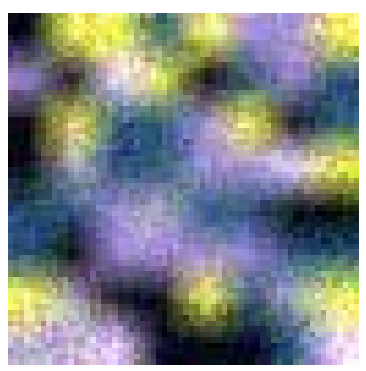

$S N R=20 \mathrm{~dB}$ 


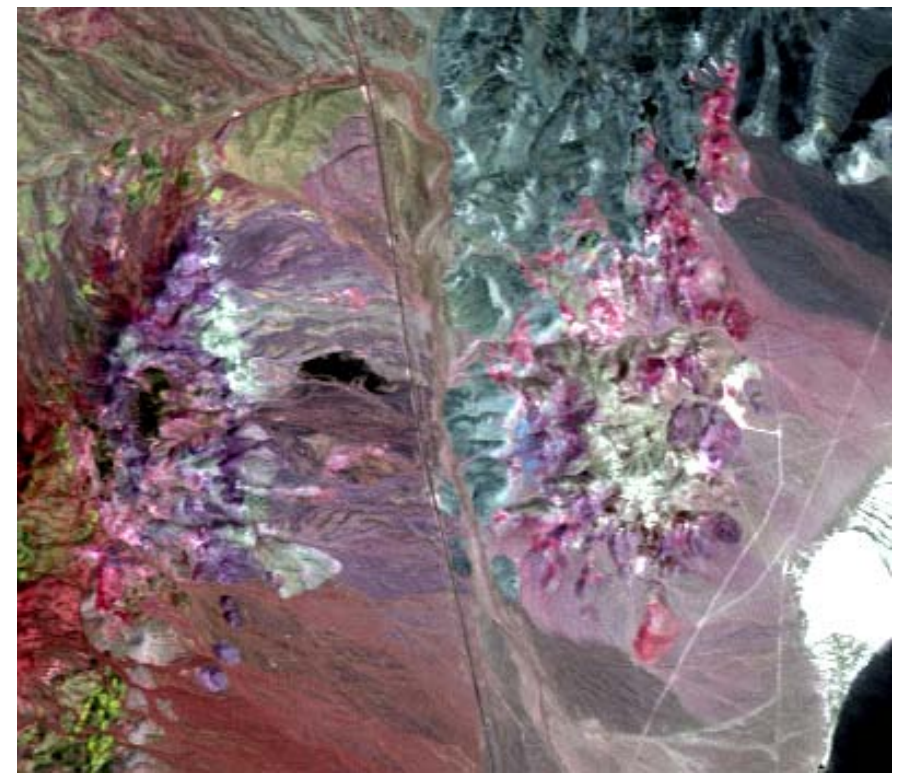

Fig. 4 False color composition of the subset of the AVIRIS Cuprite dataset (Red: Band 183, 2101.0 nm;

Green: Band 193, 2200.8 nm; Blue: Band 207, 2340.2 nm).

\subsection{Reduction of the calculation complexity}

To reduce the calculation complexity, in this study, only a part of the image pixels was used as candidate endmembers to be put in the EE-DFA. The candidate endmembers were selected based convex polyhedron. Under idealized linear condition, the endmembers form a convex simplex in the spectral space and all the image pixels lie inside this simplex, with the endmembers as geometric vertices [36]. Due to some non-linear factors and the noise, some mixed pixels are outside of the simplex, and these outside mixed pixels look like the "vertices" (endmembers) of the simplex. In fact, this is an important reason that impacts the endmember extraction results of SMACC and N-FINDR (Detailed explanation can be found in part $C$ "Experiments on the synthetic hyperspectral datasets”). Even so, the real endmembers are still near these "vertices", and it is highly impossible that the pixels that are far away from these "vertices" are the real endmembers. Hence, in this study, we selected the candidate endmembers from the pixels near the vertices of the simplex.

Here, we give a conceptual explanation on selecting candidate endmembers in 2-dimemensinal spectral space (Fig. 5). In Fig. 5, three points $A, C, D$, are actually the real endmembers and other black points are the mixed pixels. However, for endmember extraction, we cannot distinguish $A, C, D$, from the 
mixed pixels near them, because just by visual investigation, $A, C, D$, also may be the mixed pixels that are outside of the simplex (the triangle in 2-dimemensinal spectral space) due to the noise. In other words, the point $B$ (Fig. 5) is actually a mixed pixels and become outside of the triangle $A C D$ due to the noise. However, just by visual analysis, $B$ could also be regarded as an endmember, instead of $A$, and $A$ could also be regarded as a mixed pixel that are outside of the triangle $B C D$. Hence, we selected the pixels near the vertices of the simplex as candidate endmembers. For a relative pure pixel (endmember), the offset between estimated reflectance and truth reflectance comes primarily from the noise. Given the probability distribution function of Gaussian distribution, in this study we selected the pixels that obey following condition as candidate endmembers.

$$
\left|p_{i j}-v_{i j}\right|<1.96 \sigma
$$

$v_{i j}$ means the reflectance of the $j$-th band of the $i$-th vertice, and $p_{i j}$ means the reflectance of the $j$-th band of the candidate pixels near the $i$-th vertice. All the pixels that obey following condition were selected as candidate endmembers. For synthetic hyperspectral datasets in this study, the $\sigma$ was determined by the equation (12) in the revised manuscript. For AVIRIS dataset, it is shown that the ratio of signal to noise is 500:1 in SWIR band in Reference [37]. The $\sigma$ was determined by both the equation (11) and (12).The real endmembers would be determined by EE-DFA method.

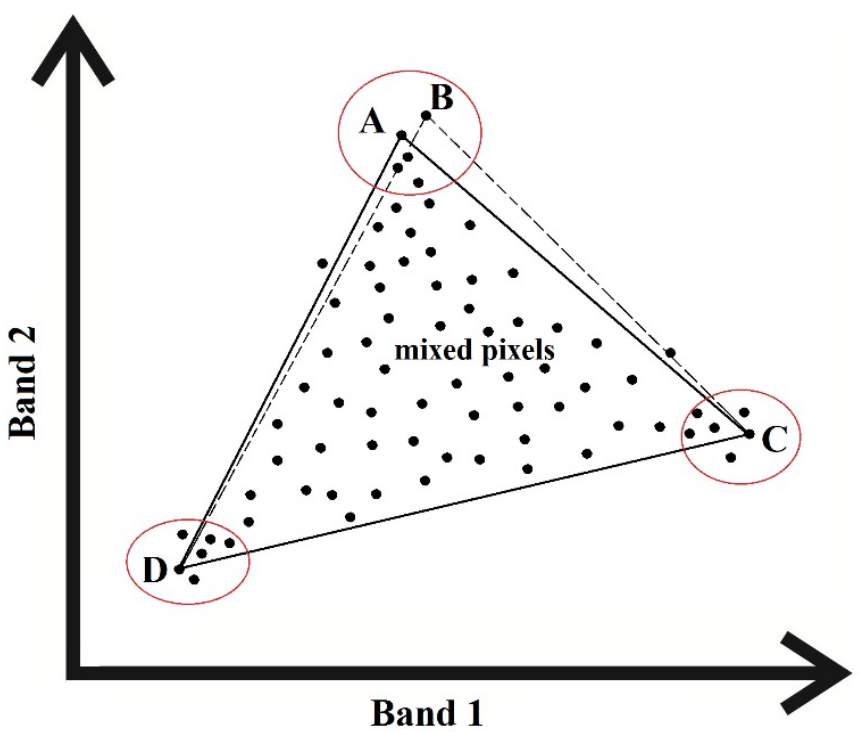

Fig. 5. Conceptual explanation on selecting candidate endmembers (black points in the red cycles) in 
For hyperspectral dataset with $L$ bands, at first, we used the Minimum Noise Fraction (MNF)

272

273

274

Transform to realize dimensionality reduction. The final dimensionality was determined by the eigenvalues and the visual quality of the MNF results. In detail, the lowest eigenvalue in the selected MNF bands should be more than 1 and the last MNF band should visually contain some useful information of ground surface, not only noise. Then the dimensionality-reduced data were used to conduct " $n$-dimensional visualization" (a function in ENVI software) to select the pixels near the vertices of the simplex as the candidate endmembers, in which $n$ could be set as the dimensionality reduced in MNF. In " $n$-dimensional visualization", all the dimensionality-reduced pixels were put in an $n$-dimensional space and the pixels near the vertices of the simplex were selected from different perspectives. In general, the number of real endmembers is much less than that of candidate endmembers. Then with the number of candidate endmembers increasing, the feasible solutions also increase. So we set the firefly population size as the number of candidate endmembers in this study.

By this way, almost all the pixels that may be real endmembers were selected. The selection steps are simple, and the result includes dozens of pixels but it is far away from the real endmember extraction result. Hence, the EE-DFA method is the key process to get the final endmembers. The purpose of this selection is only to reduce the calculation complexity of the EE-DFA. If we do not select candidate pixels with the above method, all the image pixels are regarded as the candidate endmembers, and the iteration times and the number of fireflies should be increased in the EE-DFA method. Candidate endmembers selection based convex simplex is only one method for selection. In other words, other candidate endmembers selection methods that can extract almost all the pixels similar to real endmembers, are also appropriate for the EE-DFA.

The computational complexity of EE-DFA was analyzed according to the number of all the cycles in the procedure. The computational complexities of EE-DFA, SMACC, N-FINDR, VCA, and MVC-NMF are listed in Table 1. In Table 1, $k$ is the iteration times of EE-DFA and MVC-NMF. $p$ is the number of reduced dimensions in corresponding methods. $q$ is the number of fireflies. $N$ is the number of pixels in the tested hyperspectral image. $M$ is the maximum iteration times of MVC-NMF the user set. For the N-FINDR algorithm, the range of $\eta$ is $2.3<\eta<2.9$ [7]. As shown by Fig.7, $k \approx 20$ in the EE-DFA. From the view of time complexity in the field of software engineering, computational complexities shown by 
299 Table 1 are with the polynomial way and belong to same level. What's more, $N>>p$ and $N>>q$, so the 300 computational complexities of are primarily determined by the number of pixels $(N)$. Hence, the

301

302

303

304

305

306

307

308

309

310

311

312

313

314

315

316

317

318

319

computational complexity of EE-DFA is similar to that of other methods. In consideration of the value of $\eta$ (N-FINDR), the complexity of N-FINDR is a little higher than that of other methods and similar conclusion has been achieved by Reference [7].

Table 1. The computational complexities of EE-DFA, SMACC, N-FINDR, VCA, and MVC-NMF

\begin{tabular}{cc}
\hline Algorithms & Computational Complexity \\
\hline EE-DFA & $O\left(k \cdot N \cdot q^{2}\right)$ \\
SMACC & $O\left(p^{2} \cdot N\right)$ \\
N-FINDR & $O\left(p^{\eta+1} \cdot N\right)$ \\
VCA & $O\left(p^{2} \cdot N\right)$ \\
MVC-NMF & $\geq O(50 \cdot k \cdot M)$ \\
\hline
\end{tabular}

\subsection{Parameters analysis and convergence}

According to the detailed steps of the proposed EE-DFA method (Fig. 1), the parameters to be analyzed are $\beta_{0}, \gamma$, and $\alpha$. As already mentioned in the "2 Firefly Algorithm (FA)", $\beta_{0}$ is the attractiveness at $r_{i j}=0$. For discrete optimization problem, in general, the movement distance from $\mathbf{x}_{\mathbf{i}}$ to $\mathbf{x}_{\mathbf{j}}$ is less than $\left(\mathbf{x}_{\mathbf{i}}-\mathbf{x}_{\mathbf{j}}\right)$. What's more, if ignoring the tiny randomization step (equation (3)), $\mathbf{x}_{\mathbf{i}}$ should be equal to $\mathbf{x}_{\mathbf{j}}$ after movement when $r_{i j}=0$ in discrete optimization problem. Hence, $\beta_{0}$ should be set as 1 in this study.

To set the parameters $\gamma$ and $\alpha$ on the endmember extraction, we conducted experiments of parametric effect with different values of $\gamma$ and $\alpha$ using the four synthetic datasets (with different SNR) and the real hyperspectral dataset (AVIRIS image). The endmember extraction results were evaluated by the RMSE of spectral unmixing (Fig. 6). For both $\gamma$ and $\alpha$, the test range is $[0,1]$ and the step is 0.1 in the parameter-effect experiments. In Fig. 6, the RMSE value was linearly interpolated.

For different images, the levels of RMSEs are different. To clearly show the locations of minimum RMSE and then select best ranges of $\gamma$ and $\alpha$, we set different scale of RMSE for different images in Fig. 6 because of no need to compare one subfigure of Fig. 6 to another. Fig. 6 indicated that the best ranges of 
$\gamma$ and $\alpha$ are $[0.5,1](\gamma)$ and $[0,0.3](\alpha)$, respectively, which is also the recommended parametric setting to

321 the readers. In this parametric range, the results obtained by EE-DFA is steadily better than that with

322 other parametric setting. We investigated all the experimental results and found that the minimum RMSE

323 were acquired near $(\gamma, \alpha)=(0.8,0.1)$. Hence, in subsequent experiments, we set $\gamma=0.8$ and $\alpha=0.1$.

(a)

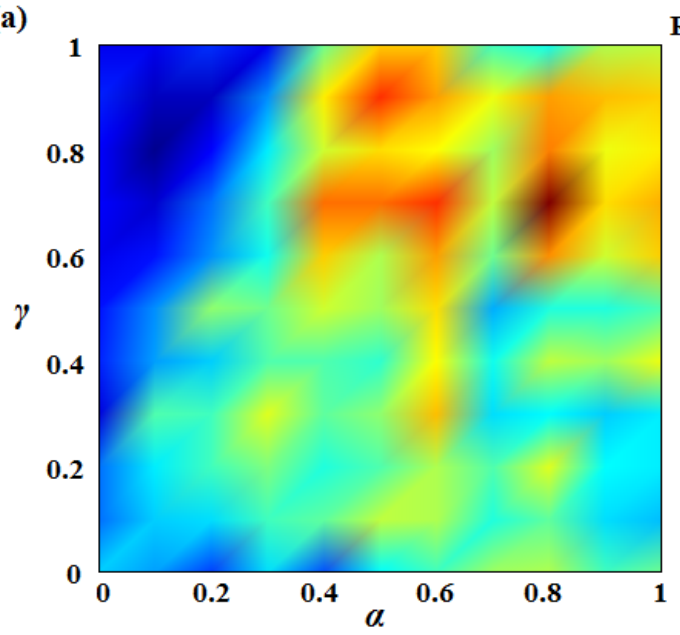

(c)

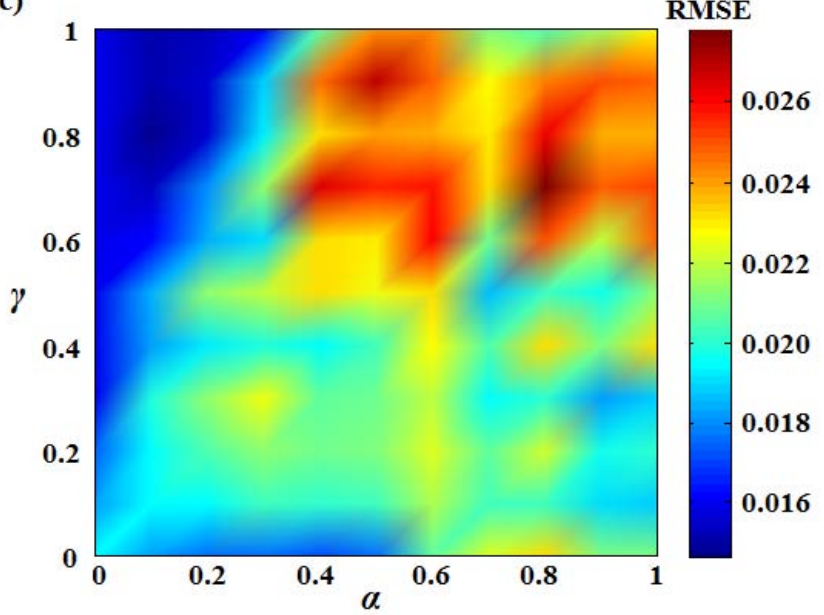

RMSE

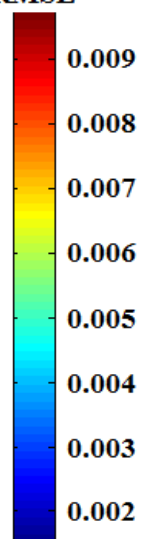

(e) (b)

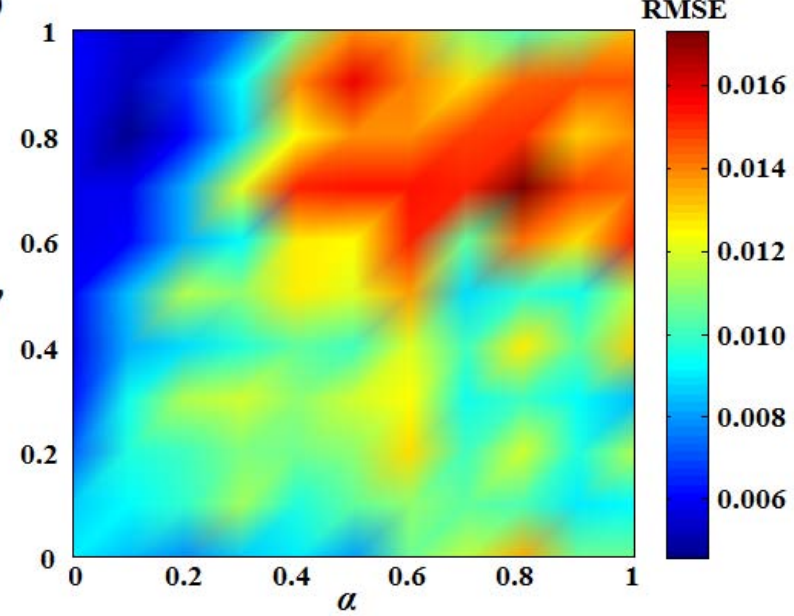

(d)

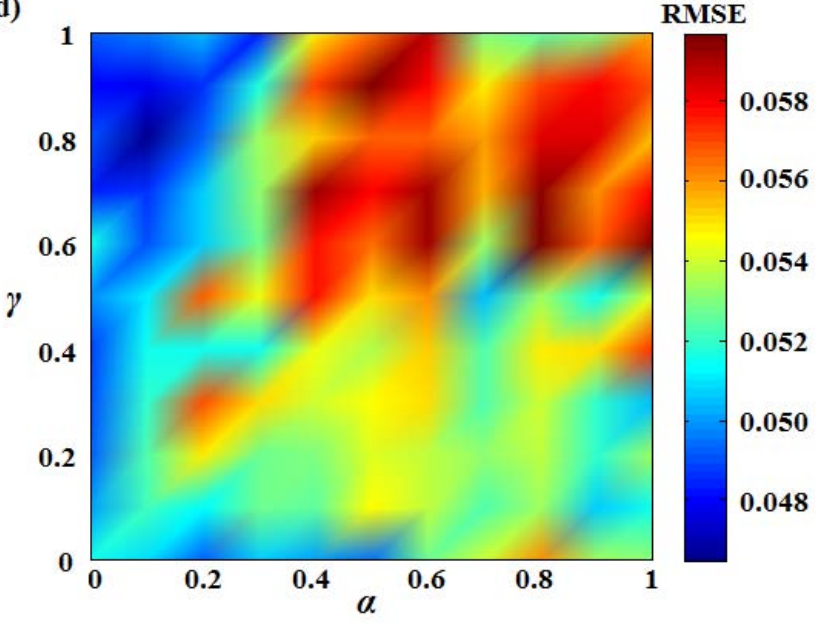

RMSE

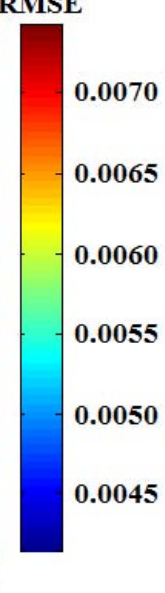

Fig. 6 The RMSE with different values of $\gamma$ and $\alpha$. (a) RMSE results of $50 \mathrm{~dB}$ synthetic hyperspectral dataset; (b) 
RMSE results of $40 \mathrm{~dB}$ synthetic hyperspectral dataset; (c) RMSE results of $30 \mathrm{~dB}$ synthetic hyperspectral dataset; (d) RMSE results of $20 \mathrm{~dB}$ synthetic hyperspectral dataset; (e) RMSE results of real hyperspectral dataset.

Here we give an explanation on the pattern indicated by Fig. 6. The characteristics of convergence and the avoidance from trapping in local optimum are of particular significance for the performance of an optimization algorithm. The parameters $\gamma$ and $\alpha$ are two important factors in determining the convergence and avoiding trapping in local optimum. As light absorption coefficient, $\gamma$ characterizes the attractiveness variation of a brighter firefly and determines how FA behaves. In condition where $\gamma$ tends to zero, the attractiveness is constant and $\beta=\beta_{0}$. In this case, the attractiveness of a firefly will not decrease with increasing the distance between two fireflies so that $\mathrm{x}_{\mathrm{i}}$ will move to the location of $\mathrm{x}_{\mathrm{j}}$ directly (equation (3)), and the objective function is easy to achieve local optimum. With increasing the value of $\gamma$, the attractiveness of a firefly tends to lower so that the fireflies fly in a more random way, and the objective function is hard to achieve convergence. The contribution of $\gamma$ are much more than that of $\alpha$ to the convergence, and for the parameter $\alpha$ in firefly algorithm, the original purpose of third term in equation (3) is to add a disturbance to help the objective function escape from local optimum [25, 27]. Since it is a disturbance, the convergence of objective function requests that this disturbance should not be too large, so the value of parameter $\alpha$ should be near zero, but could not equal zero. In this case, to some degree, the value of $\gamma$ should be away from zero. Hence, the lower value of RMSE presents in the left-up corner in subfigures of Fig. 6. The most arresting pattern is the higher RMSE in the right-up corner in the subfigures of Fig. 6. The higher both $\gamma$ and $\alpha$ lead to very random behavior of fireflies, and this corresponds to a relative random value of RMSE.

In this study, the condition that judges if the convergence of the objective function is achieved is that the iteration times are more than 100 or the updated maximum brightness Ibest keeps constant in the last three iterations (for one iteration, it starts from "Set $i=1$ ” and ends with the judgement of convergence in Fig. 1). Fig. 7 shows the convergence process of the objective function on the four synthetic datasets and a real dataset in this study, which illustrates that the objective function could achieve the convergence successfully. 


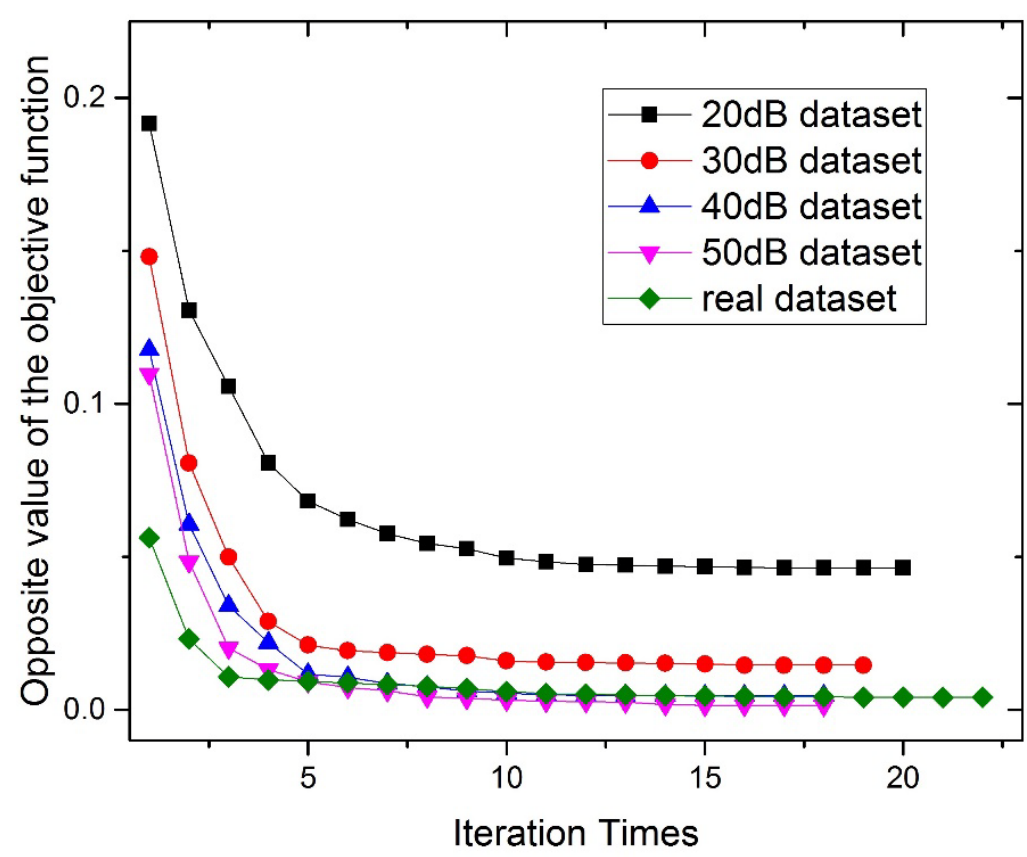

351

Fig. 7 The convergence process of the objective function on the tested datasets

\subsection{Comparative experiments on the synthetic hyperspectral datasets}

It's unrealistic that all the endmember extraction methods are used for comparative experiments. Hence, we inevitably had to make a selection from these methods, which is the nature of any paper proposing new method. Popular autonomous endmember extraction methods from hyperspectral image mainly include N-FINDR, SMACC, VCA, IEA, ORASIS etc.. The performance of N-FINDR is generally better than ORASIS when SNR is low [7]. VCA projects the image data onto a vector orthogonal to the subspace created by the endmembers already selected, and the advantage of SMACC is that SMACC replaces orthogonal projection by oblique projection to meet the non-negativity restriction of fractional abundance (equation (6)) [7] [8] [38]. IEA is to reach the minimum unmixing error based on pixel by pixel, so the calculation is complicated and IEA is less popular than N-FINDR and SMACC. What's more, it has been shown that SMACC performs better than IEA [8]. MVC-NMF is also a typical method in last recent years [15]. Hence, SMACC, N-FINDR, VCA, and MVC-NMF are selected for comparative experiments in this paper, and all the tested datasets are with their original noise.

Then we conducted experiments on all the four synthetic hyperspectral datasets and the experimental results of the proposed EE-DFA method were compared with those of four typical methods (SMACC, N-FINDR, VCA, and MVC-NMF) (Table 2 to Table 5). The performance of different 
endmember extraction methods was evaluated by two parameters: (1) the spectral angles between the extracted endmembers and the ideal endmembers, (2) the RMSE of the spectral unmixing. The definition of spectral angle $\theta$ between two spectra is shown in the equation (14), which is same with the calculation in the typical hyperspectral mapping method: Spectral Angle Mapper (SAM) [39] [40].

$$
\theta=\arccos \frac{\sum_{i=1}^{L} S_{i} T_{i}}{\sqrt{\sum_{i=1}^{L} S_{i}^{2} \sum_{i=1}^{L} T_{i}^{2}}}, \theta \in\left[0, \frac{\pi}{2}\right]
$$

$S_{i}$ and $T_{i}$ mean the reflectance of the $i$-th band of the extracted endmember spectrum and the ideal endmember spectrum, respectively. For the synthetic hyperspectral datasets, the ideal endmembers are the resampled six selected spectra from USGS library. A less spectral angle $\theta$ means more similarity between the two spectra.

To analyze the overall performance of different methods, the average of spectral angles from different methods in different SNR situations, was also calculated and shown in Table 2 to Table 5. The spectral angle of "Almandine" in SMACC method is distinctly far from the angles of other materials ( $\mathrm{SNR}=50,40,30 \mathrm{~dB}$ ), which would primarily determine the average. Hence, when calculated the average, we deleted the spectral angle of "Almandine" in SMACC method (please see the "Note" under Table 2 to Table 4). If not, the average of spectral angles would be meaningless. In fact, we found the "Almandine" spectrum in SMACC method with $\mathrm{SNR}=20 \mathrm{~dB}$ was also wrong, but the angle was not distinctly far away from other angles and thus was not deleted when the average was calculated. In all the situations (SNR=50, 40, 30, $20 \mathrm{~dB}$ ), the average of spectral angles with EE-DFA method is distinctly less than those with SMACC and N-FINDR.

Here, the extracted endmember spectra of the dataset with $\mathrm{SNR}=40 \mathrm{~dB}$ were used as an example for analysis, and were shown in Fig. 8. The comparison between Fig. 2 and Fig. 8 indicated that the endmembers extracted by EE-DFA matched well with the original real endmembers and the differences mainly come from the noise that we added. For the endmembers extracted by SMACC, in fact, the first endmember is similar to Axinite, not Almandine. Hence, two Axinite spectra were extracted by SMACC and this is the reason why the spectral angle of the "Almandine" spectra with SMACC method is abnormal (Table 3). Except the first endmember, other endmembers extracted by SMACC matched well with the original real endmembers (Fig. 2 and Fig. 8). For the endmembers extracted by N-FINDR, the 
395 first endmember is better than that by SMACC. The fifth endmember (Carnallite) is the best one among 396 all the six extracted endmembers (Table 3), and is almost same with that by EE-DFA and N-FINDR. But 397 other four endmembers are worse than those extracted by EE-DFA and N-FINDR.

398 The endmembers extracted by VCA are similar to those by EE-DFA, but both the spectral angles 399 and the unmixing RMSE with VCA are larger. In terms of the unmixing RMSE, when the SNR =20 dB, 400 the performance of MVC-NMF is better than EE-DFA (Table 5). As the SNR increases, the performance 401 of EE-DFA exceeds other methods including MVC-NMF. When the SNR=30dB, the performance of 402 MVC-NMF is similar to that of EE-DFA (Table 4), and EE-DFA outperforms MVC-NMF when $403 \mathrm{SNR}=40 \mathrm{~dB}$ and $50 \mathrm{~dB}$. In terms of spectral angles, the performance of EE-DFA is better with SNR=40 $404 \mathrm{~dB}$ and $50 \mathrm{~dB}$. When SNR=30 dB, the spectral angles with EE-DFA are also smaller for some materials 405 (e.g., Almandine and Carnallite). In other cases, the MVC-NMF performs better. Table 2. Comparison of the results of EE-DFA, SMACC, N-FINDR, VCA, and MVC-NMF methods on the synthetic hyperspectral dataset $(\mathrm{SNR}=50 \mathrm{~dB})$

\begin{tabular}{lccccc}
\hline \hline \multirow{2}{*}{ Materials } & EE-DFA & SMACC & N-FINDR & VCA & MVC-NMF \\
\cline { 2 - 6 } & \multicolumn{5}{c}{ Spectral Angles (SA) } \\
\hline Almandine & 0.0030 & 0.3762 & 0.0031 & 0.0034 & 0.0452 \\
Ammonio-Jarosite & 0.0030 & 0.0032 & 0.0296 & 0.0031 & 0.0067 \\
Antigorite & 0.0032 & 0.0033 & 0.1116 & 0.0030 & 0.0128 \\
Axinite & 0.0031 & 0.0032 & 0.1089 & 0.0032 & 0.0247 \\
Biotite & 0.0032 & 0.0031 & 0.0893 & 0.0034 & 0.0193 \\
Carnallite & 0.0032 & 0.0033 & 0.0033 & 0.0033 & 0.0476 \\
\hline Average (SA) & 0.00312 & 0.00322 & 0.05763 & 0.00323 & 0.02605 \\
RMSE & 0.0015 & 0.0083 & 0.0136 & 0.0017 & 0.0053 \\
\hline
\end{tabular}

Note: (The average of spectral angles with SMACC do not include 'Almandine')

409

Table 3. Comparison of the results of EE-DFA, SMACC, N-FINDR, VCA, and MVC-NMF methods on the synthetic hyperspectral dataset $(\mathrm{SNR}=40 \mathrm{~dB})$

\begin{tabular}{lccccc}
\hline \hline \multirow{2}{*}{ Materials } & EE-DFA & SMACC & N-FINDR & VCA & MVC-NMF \\
\cline { 2 - 6 } & \multicolumn{5}{c}{ Spectral Angles (SA) } \\
\hline Almandine & 0.0102 & 0.3774 & 0.1826 & 0.0102 & 0.0452 \\
Ammonio-Jarosite & 0.0096 & 0.0108 & 0.0575 & 0.0100 & 0.0068 \\
Antigorite & 0.0102 & 0.0101 & 0.2325 & 0.0103 & 0.0127 \\
Axinite & 0.0103 & 0.0098 & 0.1971 & 0.0109 & 0.0246 \\
Biotite & 0.0099 & 0.0105 & 0.0091 & 0.0109 & 0.0193 \\
\hline
\end{tabular}




\begin{tabular}{lccccc}
\hline Carnallite & 0.0094 & 0.0103 & 0.3466 & 0.0105 & 0.0476 \\
\hline Average (SA) & 0.00993 & 0.01030 & 0.17090 & 0.01047 & 0.02603 \\
RMSE & 0.0047 & 0.0108 & 0.0352 & 0.0048 & 0.0065 \\
\hline
\end{tabular}

412

413

414

415

416

417

418

419

420

421

422

42

424

Table 4. Comparison of the results of EE-DFA, SMACC, N-FINDR, VCA, and MVC-NMF methods on the synthetic hyperspectral dataset ( $\mathrm{SNR}=30 \mathrm{~dB}$ )

\begin{tabular}{lccccc}
\hline \hline \multirow{2}{*}{ Materials } & EE-DFA & SMACC & N-FINDR & VCA & MVC-NMF \\
\cline { 2 - 6 } & \multicolumn{5}{c}{ Spectral Angles (SA) } \\
\hline Almandine & 0.0304 & 0.3795 & 0.1288 & 0.0325 & 0.0454 \\
Ammonio-Jarosite & 0.0309 & 0.0292 & 0.1319 & 0.0309 & 0.0070 \\
Antigorite & 0.0306 & 0.0309 & 0.2548 & 0.0309 & 0.0128 \\
Axinite & 0.0295 & 0.0304 & 0.5395 & 0.0314 & 0.0249 \\
Biotite & 0.0286 & 0.0315 & 0.1404 & 0.0294 & 0.0191 \\
Carnallite & 0.0298 & 0.0321 & 0.0321 & 0.0321 & 0.0478 \\
\hline Average (SA) & 0.02997 & 0.03082 & 0.20458 & 0.03120 & 0.02617 \\
RMSE & 0.0146 & 0.0191 & 0.0514 & 0.0147 & 0.0131 \\
\hline
\end{tabular}

Note: (The average of spectral angles with SMACC do not include ‘Almandine’)

Table 5. Comparison of the results of EE-DFA, SMACC, N-FINDR, VCA, and MVC-NMF methods on the synthetic hyperspectral dataset $(\mathrm{SNR}=20 \mathrm{~dB})$

\begin{tabular}{lccccc}
\hline \hline \multirow{2}{*}{ Materials } & EE-DFA & SMACC & N-FINDR & VCA & MVC-NMF \\
\cline { 2 - 6 } & \multicolumn{5}{c}{ Spectral Angles (SA) } \\
\hline Almandine & 0.1001 & 0.3972 & 0.2296 & 0.1061 & 0.0469 \\
Ammonio-Jarosite & 0.0988 & 0.0986 & 0.1352 & 0.1081 & 0.0085 \\
Antigorite & 0.1046 & 0.3895 & 0.1446 & 0.1057 & 0.0124 \\
Axinite & 0.1018 & 0.1021 & 0.3264 & 0.1022 & 0.0243 \\
Biotite & 0.0972 & 0.0995 & 0.8797 & 0.1003 & 0.0197 \\
Carnallite & 0.0963 & 0.1029 & 0.1029 & 0.3505 & 0.0494 \\
\hline Average (SA) & 0.09980 & 0.19830 & 0.30307 & 0.14548 & 0.02687 \\
RMSE & 0.0464 & 0.0565 & 0.0657 & 0.0489 & 0.0375 \\
\hline
\end{tabular}

In detail, for same SNR and material, almost all the spectral angles with EE-DFA are the minimum when compared with SMACC and N-FINDR. Here we use the term “almost”, because only several minimum spectral angles were acquired by SMACC or N-FINDR. Even so, in these unexpected results, the spectral angle with EE-DFA is very close to the minimum value, such as 0.0032 versus 0.0031 $(\mathrm{SNR}=50 \mathrm{~dB}$, Biotite), 0.0102 versus 0.0101 (SNR=40 dB, Antigorite), 0.0103 versus 0.0098 ( $\mathrm{SNR}=40$ 
$\mathrm{dB}$, Axinite), 0.0099 versus 0.0091 (SNR=40 dB, Biotite), 0.0309 versus 0.0292 ( $\mathrm{SNR}=30 \mathrm{~dB}$, Ammonio-Jarosite), 0.0988 versus 0.0986 ( $\mathrm{SNR}=20 \mathrm{~dB}$, Ammonio-Jarosite). This flaw cannot change the fact that the performance of EE-DFA evaluated by spectral angle, is distinctly better than that of other methods.
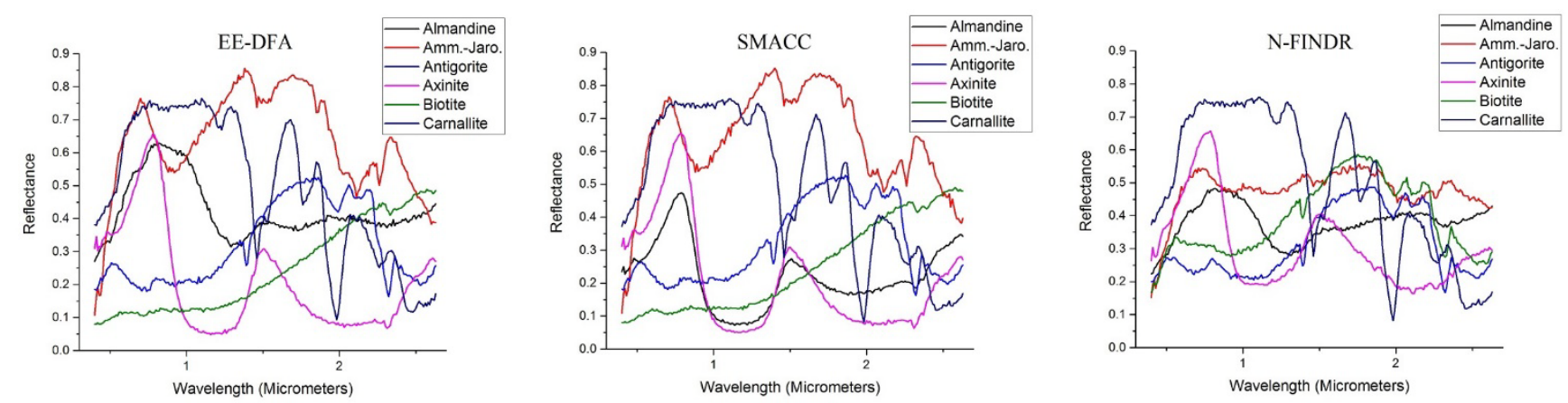

429

430

431

432

433

434

435

436

437

438

439

440

441

442

443
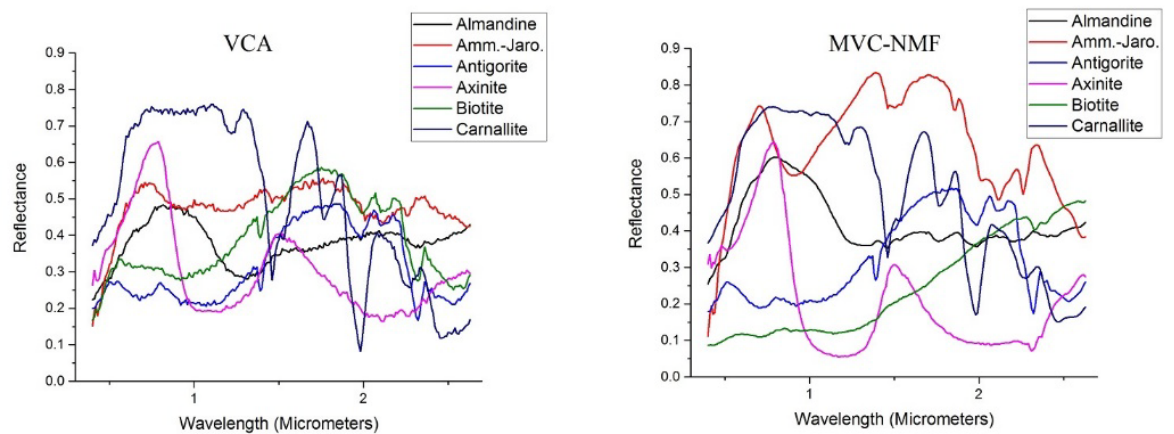

Fig. 8 The extracted endmember spectra of synthetic dataset with SNR=40 dB. (Amm.-Jaro. is the abbreviation of Ammonio-Jarosite for convenience)

In general, we can approximately regard two spectra as one material when the spectral angle $\theta<0.1$ rad. For example, the default threshold of SAM method in the ENVI software is exactly 0.1 rad. Most of spectral angles with the EE-DFA are less than $0.1 \mathrm{rad}$. The maximum spectral angle with EE-DFA is only $0.1046 \mathrm{rad}$ ("Antigorite”, $\mathrm{SNR}=20 \mathrm{~dB}$ ). In this sense, almost all the endmembers extracted by the EE-DFA method in different SNR situations were correct. However, incorrect identifications were obviously presented in the results from SMACC and N-FINDR, for example, the "Carnallite” spectrum with N-FINDR (SNR=40 dB) and all the “Almandine” spectra with SMACC method.

Both the RMSE and the average of spectral angles decreases with increasing the SNR, which is appropriate for all the methods. What's more, when the noise is high (SNR=20 dB), according to the spectral angles of different materials, the endmembers were still extracted correctly by EE-DFA, and EE-DFA has much better performance than other methods. Robustness is important for a proposed 
method [41, 42, 43]. This result demonstrates that the proposed EE-DFA method is robust and stable when the noise of the hyperspectral dataset increases sharply, which is different with SMACC and N-FINDR.

Here we give an explanation on the results acquired by EE-DFA, SMACC, N-FINDR, VCA, and MVC-NMF. As described in the “1. Introduction”, SMACC, N-FINDR, and VCA are based on a simple geometrical precondition that the endmembers are the vertices of the convex simplex in spectral space. However, due to noise and other non-linear factors, some mixed pixels are outside of the simplex and identified as vertices of the convex simplex by SMACC and N-FINDR. For example, in Fig. 5, the mixed pixel $B$ are outside of the triangle $A C D$ and looks like a vertex of another triangle $B C D$. The objective of the N-FINDR method is to find the maximum-area (or volume) simplex and the area of the triangle $A C D$ is less than the triangle $B C D$. Hence, $B$ was regarded as an endmember by N-FINDR and thus the accuracy of endmember extraction by N-FINDR decreased. Similar mechanism is appropriate for the SMACC method. This is the reason why the results of SMACC and N-FINDR are impacted apparently by the SNR. For the proposed EE-DFA, the real endmember $A$ and the mixed pixel $B$ (Fig. 5) can be separated by the RMSE of the spectral unmixing and thus better endmember extraction results can be acquired. Hence, the proposed EE-DFA method is appropriate for non-simplex in spectral space and robust under different SNR. The MVC-NMF method is to calculate the endmember signals, not to identify the relative pure pixels directly. Thus, for the unmixing RMSE, when the SNR is low (SNR=20 $\mathrm{dB}$ ), the performance of MVC-NMF is better because of the function of removing noise from real pixels. However, as the SNR increases, this advantage of MVC-NMF was not significant, and the performance of EE-DFA becomes the best of all the compared methods.

In above experiments, there are pure pixels in the synthetic datasets. To illustrate the performance of the EE-DFA when there is no pure pixels in the dataset, we fixed the SNR=30dB and generated a dataset without pure pixels. The generating method is similar to the method described in Section 4.1.1. The sole difference is: after a $9 \times 9$ averaging filter was applied, we replaced all the pure pixels with mixture pixels that are made up of all endmembers of equal abundances (i.e., in these pixels, the abundance of each endmember equals $1 / \mathrm{m}$.). The experimental results are shown in Table 6. Table 6 suggests that EE-DFA obtained smaller spectral angles and RMSE, and hence the performance of EE-DFA is still better than that of other methods (SMACC, N-FINDR, VCA). The MVC-NMF was designed for highly mixed 
473 image, hence the RMSE with MVC-NMF is less than EE-DFA. But in terms of spectral angles, the 474 performance of EE-DFA is better for some materials (e.g., Almandine and Carnallite).

Table 6 Comparison of the results of EE-DFA, SMACC, N-FINDR, VCA, and MVC-NMF methods on 477 the synthetic hyperspectral dataset without pure pixels

\begin{tabular}{lccccc}
\hline \hline \multirow{2}{*}{ Materials } & EE-DFA & SMACC & N-FINDR & VCA & MVC-NMF \\
\cline { 2 - 6 } & \multicolumn{5}{c}{ Spectral Angles (SA) } \\
\hline Almandine & 0.0295 & 0.0295 & 0.2139 & 0.0517 & 0.0526 \\
Ammonio-Jarosite & 0.0298 & 0.0411 & 0.2350 & 0.0338 & 0.0073 \\
Antigorite & 0.0377 & 0.0377 & 0.1315 & 0.0568 & 0.0137 \\
Axinite & 0.0449 & 0.0449 & 0.2237 & 0.0538 & 0.0261 \\
Biotite & 0.0441 & 0.0457 & 0.1709 & 0.0441 & 0.0217 \\
Carnallite & 0.0369 & 0.0369 & 0.0369 & 0.0523 & 0.0551 \\
\hline Average (SA) & 0.03715 & 0.03930 & 0.16865 & 0.04875 & 0.02942 \\
RMSE & 0.0155 & 0.0157 & 0.0473 & 0.0166 & 0.0135 \\
\hline
\end{tabular}

478

\subsection{Comparative experiments on the real hyperspectral dataset}

The experimental results of the EE-DFA method on the AVIRIS Cuprite dataset were also compared with those of four typical methods (SMACC, N-FINDR, VCA, and MVC-NMF) (Table 7).

482 The spectra of endmember extracted by the four methods and the relevant USGS library spectra are 483 shown in Fig. 9.

Table 7. Comparison of the results of EE-DFA, SMACC, N-FINDR, VCA, and MVC-NMF methods on the real hyperspectral dataset

\begin{tabular}{lccccc}
\hline \hline \multirow{2}{*}{ Materials } & EE-DFA & SMACC & N-FINDR & VCA & MVC-NMF \\
\cline { 2 - 6 } & & \multicolumn{4}{c}{ Spectral Angles (SA) } \\
\hline Jarosite & 0.0306 & 0.0344 & 0.0452 & 0.0928 & 0.0454 \\
Goethite & 0.0519 & 0.0766 & 0.0549 & 0.1229 & 0.0546 \\
& & & 0.0551 & & \\
Palygorskite & 0.0527 & unknown & 0.0559 & 0.0712 & 0.0733 \\
Kaolinite & 0.0461 & 0.0581 & unknown & 0.0805 & unknown \\
Alunite & 0.0446 & 0.0665 & 0.0564 & 0.1029 & 0.0980 \\
Montmorillonite & 0.0494 & 0.0629 & 0.0511 & unknown & 0.0787 \\
Calcite & 0.0630 & 0.0778 & unknown & 0.0832 & 0.1400 \\
Desert Varnish & 0.0554 & 0.0776 & 0.0609 & 0.0746 & 0.2402 \\
\hline RMSE & 0.0041 & 0.0047 & 0.0084 & 0.0090 & 0.0045 \\
\hline
\end{tabular}



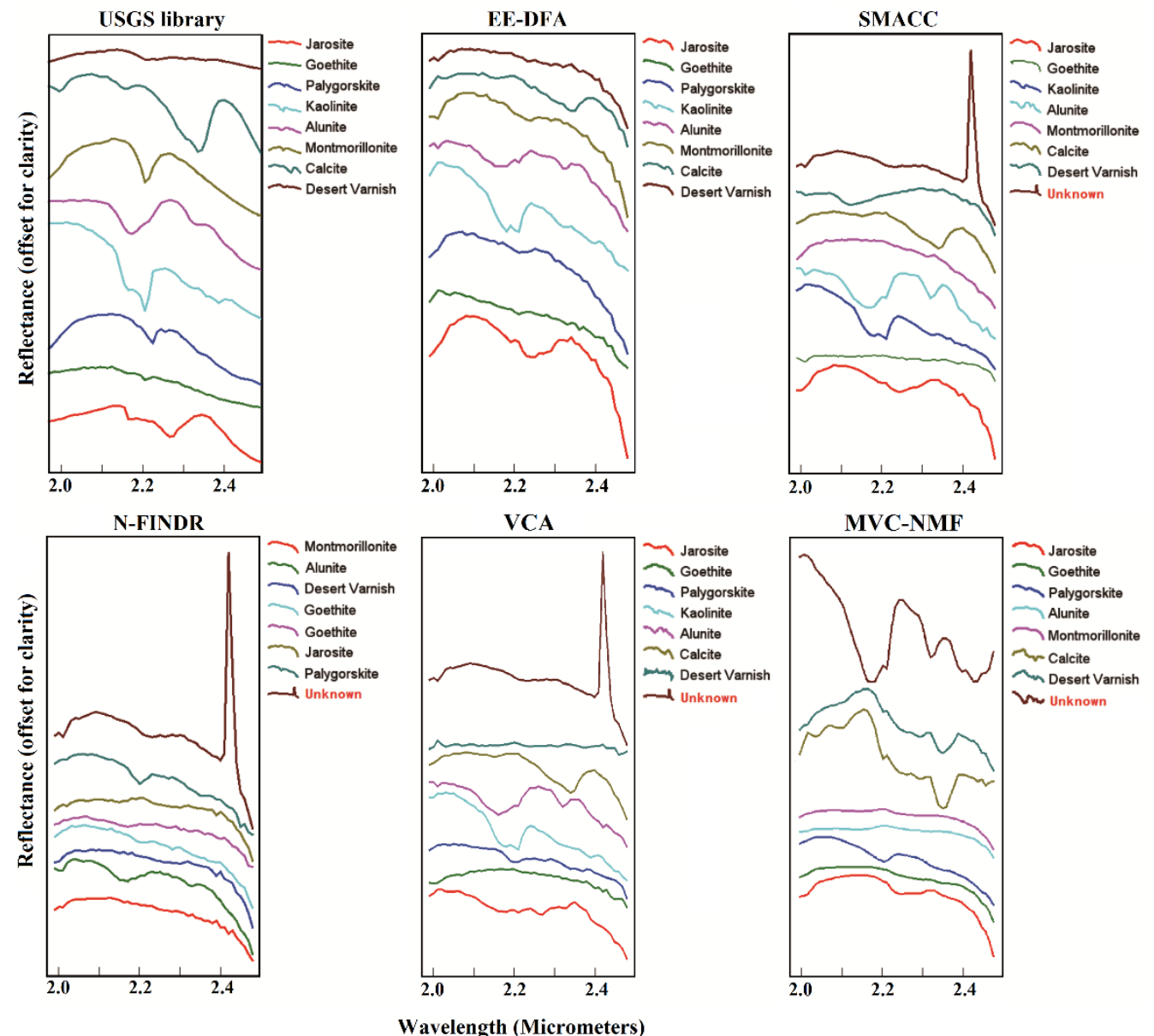

487

488

489

490

491

492

493

494

495

496

497

498

499

500

501

Fig. 9 Spectra from the USGS library and endmembers extracted by EE-DFA, SMACC, N-FINDR, VCA, and MVC-NMF from the AVIRIS image.

For the Cuprite AVIRIS subscene, in terms of RMSE, the order of different methods is EE-DFA $<$ SMACC $<$ N-FINDR (Table 7). For both SMACC and N-FINDR, there is an endmember spectrum that cannot be identified, because an abnormal high reflection was presented at approximate $2420 \mathrm{~nm}$ (Fig. 9). Moreover, there are two 'Goethite' spectra that were extracted as endmembers by N-FINDR. For all the identified endmembers, the spectral angles with EE-DFA method are minimum. In terms of both spectral angles and the unmixing RMSE, EE-DFA outperforms VCA. The unmixing RMSE of MVC-NMF is similar to that of EE-DFA (Table 7). But we investigated the endmember signals calculated by MVC-NMF and found that some of these endmembers are away from standard pure reflectance signals (Table 7 and Fig. 9). The reason is that MVC-NMF calculates new endmember signals to decrease the unmixing error during the iterations, hence the unmixing RMSE is better than those of SMACC, N-FINDR, and VCA. 
502 According to both RMSE and spectral angles, the experimental results on the AVIRIS dataset 503 demonstrate that EE-DFA can extracted endmembers successfully from hyperspectral image and is 504 better than the typical popular methods.

\section{Conclusions}

This paper proposed a novel method named EE-DFA for endmember extraction from hyperspectral 507 extraction.

Here several conclusions were reached. (1) The experiments on both synthetic and real hyperspectral images demonstrate that the endmembers extracted by EE-DFA matched the true/standard spectra better (less spectral angles), compared to the endmembers extracted by existing popular method. (2) EE-DFA reduces the influence from the non-linear factors and the noise, so better spectral unmixing results (less RMSE) can be acquired using the endmembers extracted by the proposed EE-DFA method. (3) The proposed EE-DFA method is robust when the SNR of the image decrease sharply.

Although experiments on synthetic hyperspectral datasets with different SNR and AVIRIS image have demonstrated the good performance of the EE-DFA, more hyperspectral datasets should also be used to validate the proposed method further in future research. In addition, for this method, the number of endmembers in the image is one of the inputs. So future work also will focus on improving the EE-DFA to realize endmember extraction without priori knowledge.

\section{Acknowledges}

This work was supported by the High Resolution Earth Observation System of National Science and Technology Major Project of China (No. 04-Y20A36-9001-15/17; No. 11-Y20A05-9001-15/16) and the program of National Natural Science Foundation of China (No. 41230747). The authors would like to thank the Exelis Visual Information Solutions Incorporated, Jet Propulsion Laboratory and United States Geological Survey (USGS) for providing the data used in this study.

\section{References}

[1] C. Zhang, Q. Qin, L. Chen, N. Wang, S. Zhao, and J. Hui, "Rapid determination of coalbed methane exploration target region utilizing hyperspectral remote sensing," Int. J. Coal Geol., vol. 150, pp. 19-34, 2015. 
529 [2] A. M. Filippi and R. Archibald, "Support Vector Machine-Based Endmember Extraction," IEEE Trans. 530 Geosci. Remote Sens., vol. 47, pp. 771-791, 2009.

531 [3] C. Chein-I and A. Plaza, "A fast iterative algorithm for implementation of pixel purity index," IEEE Geosci. 532 Remote Sens. Lett., vol. 3, pp. 63-7, 2006.

533 [4] Q. Qin, Z. Zhang, L. Chen, N. Wang, and C. Zhang, "Oil and gas reservoir exploration based on hyperspectral 534 remote sensing and super-low-frequency electromagnetic detection," J. Appl. Remote Sens., vol. 10, 2016.

535 [5] M. E. Winter, "N-FINDR: An algorithm for fast autonomous spectral endmember determination in 536 hyperspectral data,” in Proc. SPIE_-Imaging Spectrometry V, M. R. Descour and S. S. Shen, Eds, 1999 , vol. $537 \quad 3753$, pp. 266-275.

538 [6] R. Neville, “Automatic endmember extraction from hyperspectral data for mineral exploration,” in Proc. 21st 539 Can. Symp. Remote Sens.—4th Int. Airborne Remote Sensing Conf. Exhib., Ottawa, ON, Canada, 1999, vol. $540 \quad$ II, pp. 891-897.

541 [7] J. Nascimento and J. Dias, "Vertex component analysis: A fast algorithm to unmix hyperspectral data," IEEE 542 Trans. Geosci. Remote Sens., vol. 43, pp. 898-910, 2005.

543 [8] J. Gruninger, A. J. Ratkowski, and M. L. Hoke, “The sequential maximum angle convex cone (SMACC) 544 endmember model,” in Proc. SPIE-Algorithms and Technologies for Multispectral, Hyperspectral, and 545 Ultraspectral Imagery X, S. S. Shen and P. E. Lewis, Eds. Bellingham, WA: SPIE, 2004, vol. 5425, pp. 1-14. 546 [9] J. H. Bowles, M. Daniel, J. M. Grossman, J. A. Antoniades, M. M. Baumback, and P. J. Palmadesso, 547 “Comparison of output from ORASIS and pixel purity calculations,” in Proc. SPIE_-Imaging Spectroscopy $548 \quad$ IV, 1998, vol. 3438, pp. 148-156.

549 [10] A. Ifarraguerri and C.-I. Chang, "Multispectral and hyperspectral image analysis with convex cones,” IEEE 550 Trans. Geosci. Remote Sens., vol. 37, no. 2, pp. 756-770, Mar. 1999.

551 [11] A. Plaza, P. Martínez, R. Pérez, and J. Plaza, "Spatial/spectral endmember extraction by multidimensional 552 morphological operations,” IEEE Trans. Geosci. Remote Sens., vol. 40, no. 9, pp. 2025-2041, Sep. 2002.

553 [12] M. Berman, H. Kiiveri, R. Lagerstrom, A. Ernst, R. Dunne, and J. F. Huntington, “ICE: A statistical 554 approach to identifying endmembers in hyperspectral images,” IEEE Trans. Geosci. Remote Sens., vol. 42, no. 555 10, pp. 2085-2095, Oct. 2004.

556 [13] M. Graña, C. Hernandez, and J. Gallego, “A single individual evolutionary strategy for endmember search in 557 hyperspectral images,” Inf. Sci., vol. 161, no. 3/4, pp. 181-197, Apr. 2004. 
[14] C. Chang, C. Wu, W. Liu, and Y. Ouyang, "A new growing method for simplex-based endmember extraction algorithm,” IEEE Trans. Geosci. Remote Sens., vol. 44, no. 10, pp. 2804-2819, Oct. 2006.

[15] L. Miao and H. Qi, "Endmember extraction from highly mixed data using minimum volume constrained nonnegative matrix factorization,” IEEE Trans. Geosci. Remote Sens., vol. 45, no. 3, pp. 765-777, Mar. 2007.

[16] Plaza, G. Martin, J. Plaza, M. Zortea, and S. Sanchez, "Recent developments in spectral unmixing and endmember extraction,” in Optical Remote Sensing-Advances in Signal Processing and Exploitation Techniques. Berlin, Germany: Springer-Verlag, 2010.

[17] Zare and P. Gader, “PCE: Piecewise convex endmember detection,” IEEE Trans. Geosci. Remote Sens., vol. 48, no. 6, pp. 2620-2632, Jun. 2010.

[18] I. Chang, C. C. Wu, C. S. Lo, and M. L. Chang, "Real-time simplex growing algorithms for hyperspectral endmember extraction,” IEEE Trans. Geosci. Remote Sens., vol. 48, pt. 1, no. 4, pp. 1834-1850, Apr. 2010.

[19] Z. Bing, S. Xun, G. Lianru, and Y. Lina, "Endmember extraction of hyperspectral remote sensing images based on the ant colony optimization (ACO) algorithm," IEEE Trans. Geosci. Remote Sens., vol. 49, pp. 2635-46, 2011.

[20] Z. Bing, S. Xun, G. Lianru, and L. Yang, "Endmember Extraction of Hyperspectral Remote Sensing Images Based on the Discrete Particle Swarm Optimization Algorithm," IEEE Trans. Geosci. Remote Sens., vol. 49, pp. 4173-6, 2011.

[21] T. Chan, A. Ambikapathi, W. Ma, and C. Chi, "Robust Affine Set Fitting and Fast Simplex Volume Max-Min for Hyperspectral Endmember Extraction," IEEE Trans. Geosci. Remote Sens., vol. 51, pp. 3982-3997, 2013.

[22] X. Geng, Z. Xiao, L. Ji, Y. Zhao, F. Wang, “A Gaussian elimination based fast endmember extraction algorithm for hyperspectral imagery,” ISPRS J. Photogrammetry Remote Sens., vol. 79, pp. 211-218, 2013.

[23] Zhang and Y. Xie, "Chaos Theory-Based Data-Mining Technique for Image Endmember Extraction: Laypunov Index and Correlation Dimension (L and D)," IEEE Trans. Geosci. Remote Sens., vol. 52, pp. 1935-1947, 2014.

[24] A. Plaza, P. Martinez, R. Perez, and J. Plaza, “A quantitative and comparative analysis of endmember extraction algorithms from hyperspectral data,” IEEE Trans. Geosci. Remote Sens., vol. 42, no. 3, pp. 650-663, Mar. 2004. 
[25] J. K. Gilang and Suyanto, "Evolutionary Discrete Firefly Algorithm for Travelling Salesman Problem," Adaptive and Intelligent Systems - Second International Conference, ICAIS 2011, Klagenfurt, Austria, September 6-8, 2011. Proceedings. pp 393-403, 2011.

DOI: 10.1007/978-3-642-23857-4_38.

[26] X. S. Yang, “Firefly Algorithms for Multimodal Optimization,” In: Watanabe, O.,Zeugmann, T. (eds.) SAGA. LNCS, vol. 5792, pp. 169-178. Springer, Heidelberg, 2009.

[27] M. K. Sayadi, R. Ramezanian, and N.Ghaffari-Nasab, “A discrete firefly meta-heuristic with local search for makespan minimization in permutation flow shop scheduling problems,” Int. J. of Industrial Engineering Computations., vol. 1, pp. 1-10, 2010.

[28] X. S. Yang, “Nature-inspired Metaheuristic Algorithm,” Luniver Press (2008).

[29] D. C. Heinz and C. I. Chang, "Fully constrained least squares linear spectral mixture analysis method for material quantification in hyperspectral imagery," IEEE Trans. Geosci. Remote Sens., vol. 39, pp. 529-545, 2001.

[30] R. Rajabi and H. Ghassemian. "Spectral Unmixing of Hyperspectral Imagery Using Multilayer NMF," IEEE Geosci. Remote Sens. Lett. vol. 12, no. 1, pp.38-42, 2008.

[31] J. M. Bioucas-Dias and J. M. P. Nascimento, “Hyperspectral subspace identification,” IEEE Trans. Geosci. Remote Sens., vol. 46, no. 8, pp. 2435-2445, 2008.

[32] Zhang, B., et al., "Improvements in the Ant Colony Optimization Algorithm for Endmember Extraction from Hyperspectral Images,” IEEE J. Sel. Topics. Appl. Earth Observ. Remote Sens., 2013. 6(2SI2): p. 522-530.

[33] Dong, Y., et al., “Maximum margin metric learning based target detection for hyperspectral images,” ISPRS J. Photogrammetry Remote Sens., 108, pp. 138-150, 2015.

[34] Chein-I Chang, et al., "Linear Spectral Mixture Analysis Based Approaches to Estimation of Virtual Dimensionality in Hyperspectral Imagery,” IEEE Trans. Geosci. Remote Sens., vol. 48, no. 11, pp. 3960-3979, 2010.

[35] Lucas Drumetz, et al., "Hyperspectral Local Intrinsic Dimensionality,” IEEE Trans. Geosci. Remote Sens., vol. 54, no. 7, pp. 4063-4078, 2016.

[36] R. Heylen, D. Burazerovic and P. Scheunders, "Fully Constrained Least Squares Spectral Unmixing by Simplex Projection," IEEE Trans. Geosci. Remote Sens., vol. 49, pp. 4112-4122, 2011. 
614 [37] Fred A. Kruse et al., Comparison of AVIRIS and Hyperion for Hyperspectral Mineral Mapping. Presented at 615 the 11th JPL Airborne Geoscience Workshop, 4-8 March 2002, Pasadena, California

616 [38] Zhang, B. and Gao L., Classification and target detection of hyperspectral image. Science Press: Beijing, 6172011.

618 [39] F. A. Kruse, A. B. Lefkoff, and J. B. Dietz, "Expert system-based mineral mapping in northern death-valley, 619 California/Nevada, using the airborne visible infrared imaging spectrometer (AVIRIS)," Remote Sens. 620 Environ. vol. 44, pp. 309-336, 1993.

621 [40] S. Schneider, R. J.Murphy, A. Melkumyan, "Evaluating the performance of a new classifier - the GP-OAD: 622 A comparison with existing methods for classifying rock type and mineralogy from hyperspectral imagery,” 623 ISPRS J. Photogrammetry Remote Sens., vol. 98, pp. 145-156, 2014.

624 [41] Zheng Yuhui, Byeungwoo Jeon, Danhua Xu, Q.M. Jonathan Wu, and Hui Zhang. 2015. "Image 625 segmentation by generalized hierarchical fuzzy C-means algorithm." Journal of Intelligent and Fuzzy Systems $62628(2): 961-73$.

627 [42] Zhou, Zhili, Yunlong Wang, Q.M. Jonathan Wu, Ching-Nung Yang, and Xingming Sun. 2016. "Effective 628 and efficient global context verification for image copy detection." IEEE Transactions on Information 629 Forensics and Security, DOI: 10.1109/TIFS.2016.2601065. (In Press)

630 [43] Wenzhi Zhao, Shihong Du. "Spectral-Spatial Feature Extraction for Hyperspectral Image Classification: A 631 Dimension Reduction and Deep Learning Approach” IEEE Trans. Geosci. Remote Sens., vol. 54, no. 8, pp. $632 \quad$ 4544-4554, 2016. 